\title{
A fast model to resolve the velocity-space of fast- ion losses detected in ASDEX Upgrade and MAST Upgrade
}

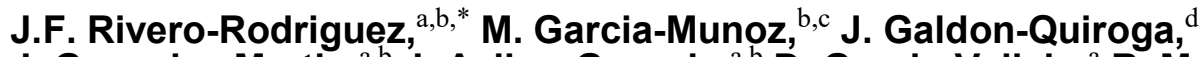 \\ J. Gonzalez-Martin, ${ }^{\mathrm{a}, \mathrm{b}}$ J. Ayllon-Guerola, ${ }^{\mathrm{a}, \mathrm{b}}$ D. Garcia-Vallejo, ${ }^{\mathrm{a}}$ R. Martin, ${ }^{\mathrm{e}}$ \\ K.G. McClements, ${ }^{e}{ }^{\mathrm{L}}$. Sanchis, ${ }^{\mathrm{b}, \mathrm{c}}$ S. Zoletnik ${ }^{\mathrm{f}}$ the ASDEX Upgrade Team, ${ }^{\mathrm{d}, * *}$ \\ the MAST Upgrade Team ${ }^{\mathrm{e}}$ and the MST1 Team \\ ${ }^{a}$ Department of Mechanical Engineering and Manufacturing, Universidad de Sevilla, \\ Camino de los Descubrimientos s/n, 41092 Seville, Spain \\ ${ }^{b}$ Centro Nacional de Aceleradores (CNA) (Universidad de Sevilla, CSIC, Junta de Andalucía) \\ ${ }^{c}$ Department of Atomic, Molecular and Nuclear Physics, Universidad de Sevilla, \\ Avenida de la Reina Mercedes s/n, 41012 Seville, Spain \\ ${ }^{d}$ Max-Planck-Institut für Plasmaphysik, \\ Garching, Germany \\ ${ }^{e}$ CCFE, Culham Science Centre, \\ Abingdon, Oxon, OX14 3DB, United Kingdom \\ ${ }^{f}$ Wigner RCP, \\ Budapest, Hungary of Institute, \\ E-mail: jeriverodus.es
}

ABSTRACT: A forward model to resolve the fast-ion loss velocity-space on a fast-ion loss detector (FILD) probe head (FILDSIM) has been extended, making it possible to perform real-time analysis of the FILD data ("real-time FILDSIM"). Parametric pre-processing with FILDSIM enables real-time mapping of the raw FILD measurements to the velocity-space of the fast-ion distribution reaching the FILD probe, which depends on the local magnetic field at the probe head. Such parametric pre-processing facilitates the study of fast-ion losses in stages of the discharge other than the flat-top, such as the ramp-up phase when changes in the local magnetic field at the probe head cannot be neglected. Real-time FILDSIM has been applied to the existing and newly installed FILDs in ASDEX Upgrade and will be used for the forthcoming FILD in MAST-Upgrade. Due to the larger size of the MAST-U FILD probe, the approximation used in FILDSIM of a uniform magnetic field in the FILD region has been generalised to the case of a non-uniform field, with gyro-orbits calculated numerically in this field.

KEYWORDS: detector; scintillator; FILD; tokamak; fast-ion; MHD; data analysis; real-time, velocity-space

\footnotetext{
${ }^{*}$ Corresponding author.

** See author list of A. Kallenbach et al. 2017 Nucl. Fusion 53102015.

*** See author list of "Dependence on plasma shape and plasma fueling for small ELM regimes in TCV and ASDEX Upgrade" by B. Labit et al. submitted to Nuclear Fusion.
} 


\section{Contents}

1. Introduction 1

2. A fast solver for the velocity-space of fast-ion losses 2

2.1 Application of the strike-map database to existing FILD measurements 3

3. Verification of the uniform magnetic field approximation 4

4. Conclusions 5

\section{Introduction}

Fast ions are the most relevant source of heating and momentum in magnetically confined fusion devices [1]. In present devices, fast-ions are produced by external heating, such as Neutral Beam Injection (NBI) and Ion-Cyclotron Resonant Heating (ICRH). In self-sustained fusion devices, $\alpha-$ particles will be responsible for upholding the necessary plasma temperatures for a burning state. In the presence of MHD activity, fast-ions are ejected due to a resonant interaction between the MHD fluctuation and the fast-ion orbits [2], leading to an increase of the fast-ion losses to the wall, which may damage plasma facing components and risk the integrity of the device. Understanding the fast-ion losses and their resonant interaction with the plasma MHD activity is crucial to reduce the impact in the performance of the fusion device and to control the MHD instabilities in the plasma. The velocity-space of the fast-ion losses is of crucial importance to study this resonant interaction, as they make possible to resolve lost ions orbits by means of backward modelling.

The scintillator-based Fast-Ion Loss Detector (FILD) [3] is a well-established diagnostic of fast-ion losses, giving local information of the fast-ion loss velocity-space, working as a magnetic spectrometer. The FILD diagnostic have been employed in the largest tokamaks, such as ASDEX Upgrade [3, 4, 5], MAST-U [6], DIII-D [7], JET [8] and NSTX [9].

In this work, the data analysis tool to resolve the velocity-space from the raw FILD measurements (FILDSIM) [10] is extended to perform a fast mapping of the data. The model is based on a parametric pre-processing of the FILD strike-map. Real-time FILDSIM is applied to the FILD systems in ASDEX Upgrade and MAST Upgrade. The work is structured as follows. In section 1, the relevance of the fast-ion loss velocity-space and its measurement with the FILD diagnostic is introduced. Section 2 describes the FILD working principle and the creation of a strike-map database to perform real-time mapping of the FILD data. This is applied offline to existing FILD measurements, showing the importance of a strike-map database in stages of the shot different to the flat-top. The validity of using a uniform magnetic field approximation in the FILD region is discussed in section 3. Section 4 summarises the features of the real-time FILDSIM and discusses future applications for this tool. 


\section{A fast solver for the velocity-space of fast-ion losses}

The FILD diagnostic, illustrated in figure 1(a), works as a magnetic spectrometer, detecting collimated fast-ions that, following their gyromotion along the tokamak magnetic field, impinge on a scintillator plate. Each collimated ion will strike the scintillator plate in a certain location according to its velocity-space (gyroradius and pitch angle), as shown in figure 1(b). A strikemap on the scintillator plate makes it possible to remap the light emitted by the scintillator plate onto velocity-space coordinates.

To construct the FILD strike-map, thousands of markers randomly distributed in position and velocity over the collimator pinhole are traced, following their gyromotion inside the FILD probe head. The gyroradius and pitch-angle of the markers that reach the scintillator plate are calculated. The strike-map is computed by calculating the strike point centroid of the markers with same gyroradius and pitch angle, resulting in a grid analogous to the one shown in figure 1(b).

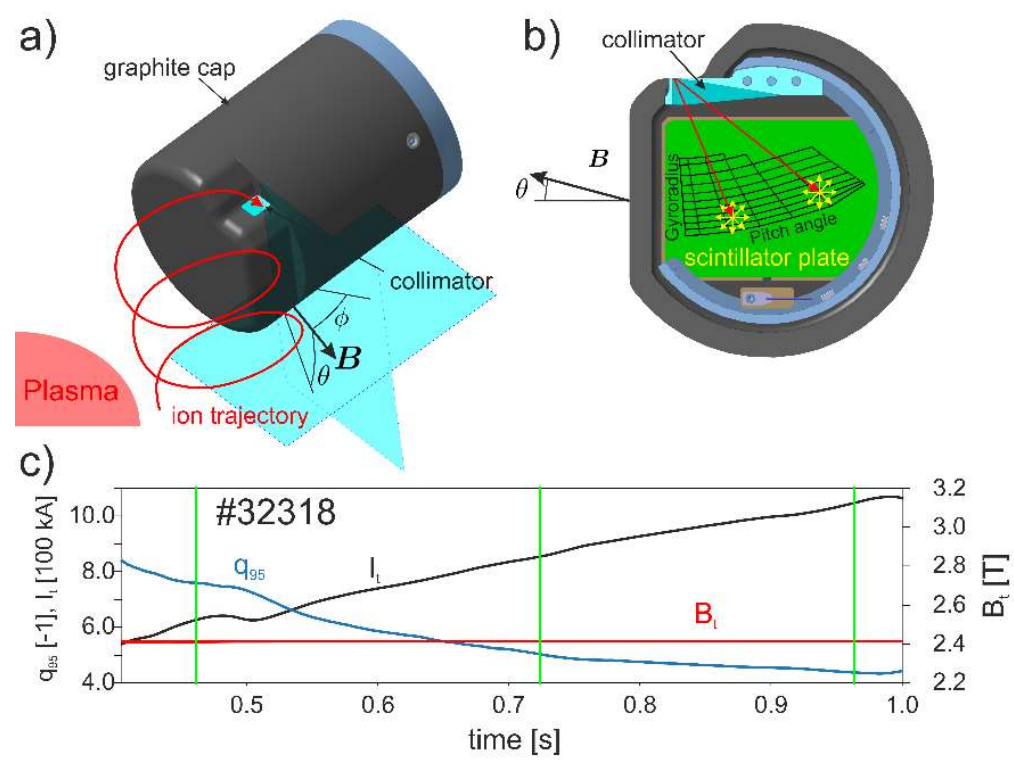

Figure 1. (a) Illustration of an escaping fast-ion orbit reaching the collimator pinhole in the FILD probe head. The two angles that define the magnetic field orientation with respect to the probe head are also shown. (b) Inner view of the FILD probe head and illustration of lost fast-ions striking the scintillator plate in different locations. (c) Evolution of the plasma current (black), toroidal magnetic field (red) and $\mathrm{q}_{95}$ (blue) during the ramp-up phase of shot \#32318 in ASDEX Upgrade.

The construction of the strike-map thus depends on the tokamak magnetic field in the FILD probe head. This initially means that different strike-maps should be computed for each different magnetic field equilibrium. However, if the magnetic field in the probe head is considered uniform, the construction of the strike-map would only depend on the orientation of the magnetic field vector with respect to the FILD probe head, that can be described by an angle in the horizontal plane, $\phi$, and an angle in the vertical plane, $\theta$, as shown in figure 1(a). A parametric pre-processing computing the strike-maps in a range along these two angles $(\phi, \theta)$ is carried out to create a database of strike-maps.

During a discharge, a camera records a sequence of images of the FILD scintillator plate. The angles $(\phi, \theta)$ are calculated in a frame-by-frame basis using the magnetic field vector provided by a real-time Grad-Shafranov solver [11]. The nearest strike-map in the database is used to map the velocity-space from the camera frame, thus giving real-time measurements of the fast-ion loss 
velocity-space. The conventional sampling frequency of the FILD cameras is $50 \mathrm{~Hz}$, thus limiting the time available to compute the fast-ion loss velocity-space to $20 \mathrm{~ms}$, which is achieved with this method. The real-time Grad-Shafranov solver can provide an estimation of the magnetic field vector in a much shorter time, $0.6 \mathrm{~ms}$.

This approach is not only relevant in the execution of real-time analysis of the FILD measurements. It also provides a reliable tool to study the FILD measurements when changes in the magnetic field may be playing an important role. This is the case of the ramp-up stage of a tokamak discharge, relevant in fast-ion studies such as the investigation of fast-ion losses driven by RSAEs [12]; the ASDEX Upgrade FILD array, where different FILDs are placed in different locations operating simultaneously; the MAST-U FILD, where the magnetic field orientation will vary significantly during a discharge; and the FILD4 radial scan [5], where fast changes in the radial position may lead to variations in the orientation of the magnetic field.

\subsection{Application of the strike-map database to existing FILD measurements}

The adaptive strike-maps can also be applied offline to existing FILD measurements. As an example, it is applied here to the ramp-up phase in shot \#32318 from ASDEX Upgrade. This is the initial phase of the discharge when the plasma current grows while the toroidal magnetic field is constant, causing drastic changes in the $\mathrm{q}_{95}$, as shown in figure 1(c). In this shot, injector 8 was active from an early stage of the discharge $(t=0.2 \mathrm{~s})$ and remained active during the entire experiment, producing a steady population of fast-ions. A spot could be observed in the FILD camera during the discharge, which does not reveal any MHD activity in a Fourier analysis. Therefore, it can be considered that this spot is due to the NBI birth prompt losses from injector 8. During the ramp-up phase, the $\mathrm{q}_{95}$ value gradually dropped and, consequently, the magnetic field orientation was changing in the outer region of the plasma, including the scrape-off layer, where the FILD is located. The FILD raw signal was observed and analysed in 3 time instants of the ramp-up phase $\left(t_{1}=0.46 \mathrm{~s}, t_{2}=0.72 \mathrm{~s}, t_{3}=0.96 \mathrm{~s}\right)$, marked with vertical lines in figure $1(\mathrm{c})$, following two different approaches.

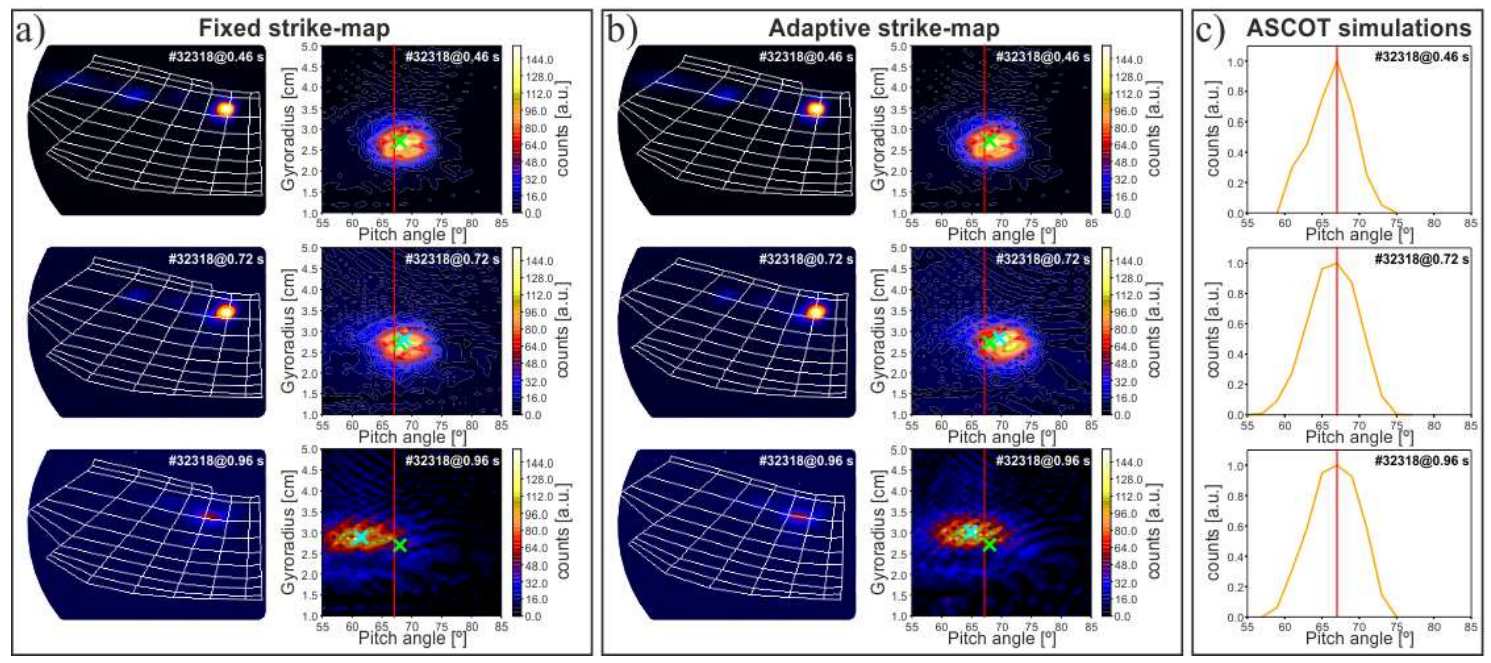

Figure 2. (a) AUG FILD raw measurements (left column) and remapping in velocity-space coordinates (right column) using a fixed strike-map. (b) AUG FILD raw measurements (left column) and remapping in velocity-space coordinates (right column) using adaptive strike-maps. The green crosses represent the centroid of the signal in $t_{1}$ while the cyan crosses represent the centroid of the signal in the corresponding time instant. (c) Pitch angle distribution of the fast-ion losses colliding with the FILD estimated by orbit following simulations performed using ASCOT. The red lines represent the pitch-angle value of the maximum in the fast-ion loss distributions. 
In the first case (figure 2(a)), a single strike-map, corresponding to the magnetic field orientation in $\mathrm{t}_{1}\left(\phi_{1}=-0.4^{\circ}, \theta_{1}=6.3^{\circ}\right)$, was computed for the 3 time instants. The mapping in velocity-space coordinates shows that the spot centroid drifted in pitch-angle from $68^{\circ}$ at $t_{1}$ to $61^{\circ}$ at $t_{3}$. The gyroradius remained relatively constant around $2.8 \mathrm{~cm}$ at the 3 time instants.

In the second case (figure 2(b)), different strike-maps, adapted to the magnetic field orientation, were used at each time instant, being collected from the strike-map database. The angles between the magnetic field and the probe head are $\left(\phi_{1}=-0.4^{\circ}, \theta_{1}=6.3^{\circ}\right),\left(\phi_{2}=-0.8^{\circ}\right.$, $\left.\theta_{2}=4.3^{\circ}\right)$ and $\left(\phi_{3}=-0.2^{\circ}, \theta_{3}=2.9^{\circ}\right)$ at each time instant, respectively. In this case, the remapping in velocity-space coordinates shows that the drift in the pitch-angle direction is reduced to a range from $68^{\circ}$ at $t_{1}$ to $65^{\circ}$ at $t_{3}$. In a similar way to the first case, the gyroradius remains almost constant in the 3 time instants.

To resolve the existence of the observed drift in the pitch-angle direction, orbit following simulations were carried out with the Monte-Carlo code, ASCOT [13]. This code produces an NBI birth fast-ion distribution and carries out forward simulations using the leap frog method described in section 3.1 of reference [13]. The velocity-space distribution of the fast-ions colliding with the FILD probe is used as an estimate of the fast-ion velocity-space measured in FILD. Simulations were carried out for the equilibrium at each of the 3 time instants. The results, shown in figure 2(c), indicate that the fast-ion loss distribution pitch-angle remained steady at the 3 time instants. The maximum value of the fast-ion distribution is at a pitch angle value of $67^{\circ}$ at all 3 time instants. This maximum value is marked with a red vertical line in the simulations and the remapped signals on figure 2 .

The results suggest that the apparent drift in the remapped signal in the case of a fixed strikemap does not have any physical cause. It is due to a drift in the FILD raw data caused by a change in the local magnetic field orientation, which is corrected using the adaptive strike-map approach.

\section{Verification of the uniform magnetic field approximation}

The construction of the strike-map database that enables the real-time analysis of the FILD data is based on the approximation of a uniform magnetic field, thus constant Larmor radius, in the FILD probe head. This approximation should be validated because, in tokamaks, the gradient of the toroidal magnetic field is proportional to $1 / \mathrm{R}^{2}$, where $\mathrm{R}$ is major radius. This is especially relevant in MAST-U where, due to its lower aspect ratio, the FILD probe head is located at a lower major radius, $\mathrm{R}=1.5 \mathrm{~m}$ in MAST-U against $\mathrm{R}=2.2 \mathrm{~m}$ in AUG, so the variation of Larmor radius across the probe head will be higher in MAST-U than in AUG.

Full orbit simulations with ASCOT, combined with the FILDSIM data analysis tools make it possible to construct a strike map using an axisymmetric magnetic field equilibrium for the MAST-U FILD. Markers are traced from the collimator to the scintillator plate with fixed gyroradius and pitch angle to construct the strike-map, as described in section 2. The FILD model is conveniently placed and oriented in its inserted position at the MAST-U grid.

At the same time, the uniform magnetic field approximation is used in FILDSIM to produce a strike-map that can be compared. The magnetic field orientation is extracted from the axisymmetric field, giving the following orientation angles $\left(\phi=-7.4^{\circ}, \theta=0.0^{\circ}\right)$.

The strike-maps made in a uniform and a non-uniform magnetic field can be observed in figure 3(a). Slight differences are observed in the gyroradius direction, as illustrated in figure 3(b) where the difference in gyroradius between the two strike maps are shown. The differences in the pitch-angle direction become larger at lower pitch-angle values, as can be seen in figure 3(c), stretching up to $2.5^{\circ}$ at a pitch-angle value of $30^{\circ}$. This might be due to the implicit consideration 

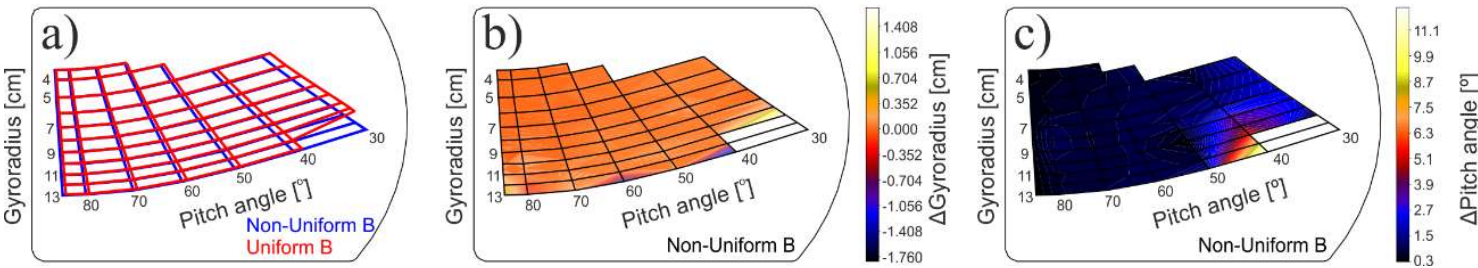

Figure 3. (a) Comparison between the MAST-U FILD strike-map constructed with a non-uniform magnetic field using ASCOT and FILDSIM (blue) and with a uniform magnetic field using FILDSIM (red). Difference in gyroradius (b) and pitch angle (c) between the uniform magnetic field and the nonuniform magnetic field strike-maps, shown over the non-uniform magnetic field strike-map.

of straight field lines in the uniform magnetic field approximation, whereas the axisymmetric field generalization grants the magnetic field curvature in the toroidal direction. Higher differences than the ones discussed can be observed in figures 3(b) and 3(c), concentrated on the strike-map borders. This is caused by slight mismatches in the strike-map borders, leading to misrepresented differences. The maximum distance between equivalent nodes in the strike-maps, i.e. same gyroradius and pitch angle, is $0.31 \mathrm{~cm}$.

It is concluded that the uniform magnetic field approximation is valid for the MAST-U FILD. Only small differences can be observed in the pitch-angle coordinate, up to $2.5^{\circ}$. As described in Section 2, the uniform magnetic field approximation makes it possible to use the strike-map database that covers the orientation of the magnetic field vector with respect to the FILD probe head $(\phi, \theta)$. Therefore, real-time velocity-space measurements will also be possible in MAST-U. The validation for the AUG FILDs was previously considered. Due to the smaller FILD probe head and the larger major radius location, the uniform approximation leads to negligible differences in the gyroradius and the pitch-angle directions. The maximum observed distance between equivalent nodes is $0.3 \mathrm{~mm}$.

\section{Conclusions}

A real-time analysis of the fast-ion loss velocity-space from the raw FILD measurements is achieved by means of a parametric database of FILD strike-maps. This new model makes it possible to accurately resolve in real-time and offline the velocity-space of the fast-ion losses when changes in the magnetic field at the FILD region cannot be neglected. The construction of a strike-map database rests on the approximation of a uniform magnetic field in the FILD region, which is validated in this work by a comparison with the non-uniform approach in the MAST-U FILD.

These new functionalities are added and combined with the existing FILDSIM features in a new graphical user interface (GUI), with the aim of unifying the data analysis of the existing and upcoming FILDs. New tomographic methods are being investigated to achieve on-the-fly tomographic reconstruction of the signal, following the same principles of this work.

The accomplishment of real-time fast-ion loss velocity-space measurements does not only accelerate the analysis of the FILD data. In future works, this information can be also used as an input to the real-time control of the FILD positioning [14] and the active control of MHD events $[15]$.

\section{Acknowledgments}

This research received funding from the V Plan Propio de Investigación de la Universidad de Sevilla (PP2016-7145). 
This work has been carried out within the framework of the EUROfusion Consortium and has received funding from the Euratom research and training programme 2014-2018 and 20192020 under grant agreement No 6333053. The views and opinions expressed herein do not necessarily reflect those of the European Commission.

\section{References}

[1] A. Fasoli et al., Chapter 5: Physics of energetic ions, 2007 Nucl. Fusion 47 S264.

[2] M. Garcia-Munoz et al., Convective and Diffusive Energetic Particle Losses Induced by Shear Alfvén Waves in the ASDEX Upgrade Tokamak, 2010 Phys. Rev. Lett. 104185002.

[3] M. Garcia-Munoz et al., Scintillator based detector for fast-ion losses induced by magnetohydrodynamic instabilities in the ASDEX upgrade tokamak, 2009 Rev. Sci. Instrum. 80 053503 .

[4] J. Gonzalez-Martin et al., First measurements of a scintillator based fast-ion loss detector near the ASDEX Upgrade divertor, 2018 Rev. Sci. Instrum. 89101106.

[5] J. Gonzalez-Martin et al., First measurements of a magnetically driven fast-ion loss detector on ASDEX Upgrade, 2019 submitted to JINST as a proceeding contribution to the $3^{\text {rd }}$ European Conference on Plasma Diagnostics.

[6] J.F. Rivero-Rodriguez et al., A rotary and reciprocating scintillator based fast-ion loss detector for the MAST-U tokamak, 2018 Rev. Sci. Instrum. 89101112.

[7] R. K. Fisher et al., Scintillator-based diagnostic for fast ion loss measurements on DIII-D, 2010 Rev. Sci. Instrum. 81 10D307.

[8] S. Baeumel et al., Scintillator probe for lost alpha measurements in JET, 2004 Rev. Sci. Instrum. 75 3563.

[9] D. S. Darrow et al., Scintillator based energetic ion loss diagnostic for the National Spherical Torus Experiment, 2008 Rev. Sci. Instrum. 79023502.

[10] J. Galdon-Quiroga et al., Velocity-space sensitivity and tomography of scintillator-based fast-ion loss detectors, 2018 Plasma Phys. Control. Fusion 60105005.

[11] L. Giannone et al., A data acquisition system for real-time magnetic equilibrium reconstruction on ASDEX Upgrade and its application to NTM stabilization experiments, 2013 Fusion Eng. Des. 88 3299-3311.

[12] M. Garcia-Munoz et al., Fast-ion transport induced by Alfvén eigenmodes in the ASDEX Upgrade tokamak, 2011 Nucl. Fusion 51103013.

[13] E. Hirvijoki et al., ASCOT: Solving the kinetic equation of minority particle species in tokamak plasmas, 2015 Comput. Phys. Commun. 185 1310-1321.

[14] J. Ayllon et al., A fast feedback controlled magnetic drive for the ASDEX Upgrade fast-ion loss detectors, 2016 Rev. Sci. Instrum. $8711 \mathrm{E} 705$.

[15] M. Garcia-Munoz et al., Active control of Alfvén eigenmodes in magnetically confined toroidal plasmas, 2019 Plasma Phys. Control. Fusion 61054007. 